\title{
PLOD2-driven IL-6/STAT3 signaling promotes the invasion and metastasis of oral squamous cell carcinoma via activation of integrin $\beta 1$
}

\author{
KEN SAITO $^{1}$, AYAKA MITSUI ${ }^{1}$, I. WAYAN SUMARDIKA ${ }^{2}$, YUSUKE YOKOYAMA ${ }^{3}$, \\ MASAKIYO SAKAGUCHI $^{4}$ and EISAKU KONDO ${ }^{1}$
}

\begin{abstract}
${ }^{1}$ Division of Molecular and Cellular Pathology, Niigata University Graduate School of Medical and Dental Sciences, Niigata 951-8510, Japan; ${ }^{2}$ Faculty of Medicine, Udayana University, Denpasar, Bali 80232, Indonesia; ${ }^{3}$ Department of Otolaryngology, Head and Neck Surgery, Niigata University Graduate School of Medical and Dental Sciences, Niigata 951-8510; ${ }^{4}$ Department of Cell Biology, Okayama University Graduate School of Medicine, Dentistry and Pharmaceutical Sciences, Okayama 700-8558, Japan
\end{abstract}

Received December 17, 2020; Accepted March 31, 2021

DOI: $10.3892 /$ ijo.2021.5209

\begin{abstract}
We previously reported that high expression of procollagen-lysine 2-oxoglutarate 5-dioxygenase 2 (PLOD2) leads to stabilization and plasma membrane translocation of integrin $\beta 1$ to promote the invasion and metastasis of oral squamous cell carcinoma (SCC). The present study aimed to further understand the relationship between PLOD2-integrin $\beta 1$ signaling and the tumor microenvironment. This study provided further advanced insights indicating that elevated interleukin (IL)-6 in the tumor microenvironment acts as a key molecule that triggers PLOD2-integrin $\beta 1$ axis-derived acceleration of tumor invasion and metastasis. It was found using the dual-luciferase reporter assay system that signal transducer and activator of transcription 3 (STAT3) activation by IL-6 was essential for increasing the expression levels of PLOD2 through direct activation of the PLOD2 promoter in oral SCC, whereas IL-6 stimulation did not contribute to integrin $\beta 1$ expression or the subsequent maturation process towards a functional form on the plasma membrane. Furthermore, the expression of IL-6 in oral SCC tissues was mainly observed in the tumor stroma. Finally, with double immunofluorescence staining, it was found that IL-6 expression occurred in CD163-positive M2 macrophages distributed around the tumor nest. These results combined with our previous results indicate that as IL-6 significantly increases STAT3-mediated PLOD2 promoter activity, IL-6 released by M2-type tumor-associated macrophages is a crucial factor
\end{abstract}

Correspondence to: $\mathrm{Dr}$ Ken Saito, Division of Molecular and Cellular Pathology, Niigata University Graduate School of Medical and Dental Sciences, 1-757 Asahimachi-dori, Chuo-ku, Niigata 951-8510, Japan

E-mail: kens@med.niigata-u.ac.jp

Key words: procollagen-lysine 2-oxoglutarate 5-dioxygenase 2, squamous cell carcinoma, invasion, integrin $\beta 1$, interleukin- 6 that promotes PLOD2-integrin $\beta 1$ axis-enhanced invasion and metastasis of oral SCC cells.

\section{Introduction}

Squamous cell carcinoma (SCC) arising from the oral cavity mucosa, tongue, gingiva and pharynx is the most common type of oral cancer. There are still a number of critical issues in the treatment of SCC, such as frequent metastasis to head/neck regions, including lymph nodes and lungs at the advanced stages, and local recurrence (1). Treatments based on the novel molecular findings of these SCCs are yet to be elucidated (2). Therefore, it is necessary to focus on the development of potential therapeutic targets to overcome cancer progression, invasiveness and metastasis.

Hydroxylation of substrates catalyzed by 2-oxoglutarate-dependent oxygenases is an essential reaction for biological processes, such as transcription, splicing, translation and protein stability (3-5). Notably, during tumor progression, research has focused on the prolyl 4-hydroxylase family for collagen hydroxylation $(6,7)$, hypoxia-inducible factor-1 $\alpha$ (HIF-1 $\alpha)$ stabilization (8-10) and the lysine demethylase family for histone demethylation $(11,12)$.

Procollagen-lysine 2-oxoglutarate 5-dioxygenase 2 (PLOD2) is a member of the PLOD family, which is involved in the lysyl hydroxylation of collagen molecules, the only target molecule identified to date. PLOD2 is considered to be an essential enzyme for the crosslinking reaction between collagen molecules outside the cell $(13,14)$. Furthermore, previous studies revealed that $P L O D 2$ is transcriptionally regulated by activation of HIF- $1 \alpha$ under hypoxic conditions and recruitment of specificity protein 1 (Sp1) and mothers against decapentaplegic homolog 3 (SMAD3) following transforming growth factor- $\beta 1$ (TGF-1 $\beta$ ) stimulation in the tumor stroma, cancer cells, sarcoma and skin fibroblasts (15-18). PLOD2 in breast cancer cells is also upregulated by adipocyte-derived interleukin (IL)-6 and leptin (19). Additional studies have reported that the promoter region of PLOD2 contains the 
binding sites of transcriptional factors, such as HIF-1 $\alpha$, SMAD3 and signal transducer and activator of transcription 3 (STAT3) (14). HIF-1 $\alpha$ - and STAT3-induced PLOD2 expression have been demonstrated in the murine PLOD2 promoter-reporter system (14). Therefore, PLOD2 expression is modulated by the activation of several transcription factors in the tumor microenvironment (TME) $(14,17,18)$.

Invasion and metastasis of cancer cells is affected by changes in the TME, which includes extracellular matrix (ECM) molecules, such as collagens (20). The TME is composed of numerous types of cells, including stromal fibroblasts, endothelial cells, immune cells and myeloid cells. These cellular communications via cell-cell attachments or soluble secretory factors, such as growth factors, cytokines and chemokines, promote a variety of cellular events, growth, migration, invasion and metastasis of cancer cells. Among TME cells, CD163-positive M2-type tumor-associated macrophages (TAMs) tend to increase in various cases of oral SCC tissues at the advanced malignant stages (21). According to the increased number of TAMs, the levels of TAM-mediated specific cytokines and chemokines will increase, and therefore abundant secretory factors from TAMs in the TME contribute to rearrangement of the tumor matrix architecture and tumor progression (22). In addition to TAMs, numerous types of TME cells may potentially modify the tumor matrix architecture and promote tumor progression. Several experimental contexts imitate the TME (including co-culture systems composed of hepatocellular carcinoma cells and cancer-associated fibroblasts; breast cancer cells and adipocytes) and induce aggressive invasion of each type of co-cultured cancer cells via upregulation of PLOD2 in the cancer cells $(14,19)$. The results suggest that soluble factors produced in the co-cultures induce PLOD2 together with extracellular matrices so that integrin $\beta 1$ on cancer cells becomes activated (23), eventually leading to increased invasiveness through an active interaction with the enriched collagen matrices in the extracellular space. The soluble factors that cause PLOD2 induction in cancer cells at significant levels are unknown. Inflammatory cytokines, such as IL- 6 and IL-8, are detected in both the saliva and serum of patients with oral cancer, and are thus potential biomarkers to diagnose oral cancer $(24,25)$. In addition to cytokines, numerous types of cancer, including oral SCC exhibit high expression of chemokine (CC motif) ligand 2 (CCL2) $(26,27$ ). However, the relationship between PLOD2-mediated tumor invasion and metastasis, and TME factors such as IL-6, IL-8 and CCL2 in refractory cancers, including those of the oral cavity and throat, remains unclear.

The present study focused on elucidating the unidentified induction machinery of PLOD2 in the TME and its role in oral cancer cells. The data indicated the possibility that targeting the identified signaling axis, IL-6-STAT3-PLOD2-integrin $\beta 1$, may have clinical utility for therapeutic regulation of oral cancer metastasis.

\section{Materials and methods}

Cell lines and cell culture. The human oral cancer cell lines (HSC-2, HSC-3, Ca9-22) used in this study were purchased from the Japanese Collection of Research Bioresources Cell Bank. Cells were maintained in RPMI-1640 medium
(FUJIFILM Wako Pure Chemical Corporation) containing $10 \%$ fetal bovine serum (FBS; HyClone; Cytiva) with $100 \mathrm{U} / \mathrm{ml}$ penicillin and $0.1 \mathrm{mg} / \mathrm{ml}$ streptomycin (Thermo Fisher Scientific, Inc.) at $37^{\circ} \mathrm{C}$ in $5 \% \mathrm{CO}_{2}$. PLOD2-knockout (PLOD2-KO) HSC-2 cell clones were generated with clustered regularly interspaced short palindromic repeats (CRISPR)/CRISPR associated protein 9 (Cas9)-based genome engineering technology as previously described (23), which were compared to the intact parental HSC-2 cells as the control [PLOD2-wild-type(PLOD2-WT)].Briefly,the23-base ofgRNA (5'-CCAGGATAATGATGATGATCAGC-3') containing the sequence of protospacer adjacent motif for PLOD2 targeting sequence (5'-GCTGATCATCATCATTATCC-3') was determined from the CRISPRdirect website (http://crispr.dbcls.jp/). The target site corresponded to the Exon 5 of PLOD2 (latest NCBI RefSeq number, NM_000935.3), which located the $\mathrm{N}$-terminal protein domain of unknown function. The plasmids hCas9 (cat. no. 41815) and gRNA Cloning Vector (cat. no. 41824) were purchased from Addgene, Inc. The genotype of PLOD2-KO cells was confirmed by Sanger DNA sequencing using ABI BigDye ${ }^{\mathrm{TM}}$ Terminator v3.1 Cycle Sequencing Kit and 3130 Genetic Analyzer (Applied Biosystem; Thermo Fisher Scientific, Inc.) according to the manufacturer's instructions. The type of mutations induced by PLOD2 knockdown was an insertion mutation (frameshift mutation disrupting open reading frames) and heterozygous mutant. The stable clone expressing green fluorescence protein was selected in $0.4 \mathrm{mg} / \mathrm{ml}$ geneticin (G418; Thermo Fisher Scientific, Inc.).

To identify possible cross-contamination with other cell lines, short tandem repeat (STR) analysis of oral cancer cells used in this study was performed by Takara Bio, Inc. Results confirmed that these cells were the same as the cells registered in Japanese Collection of Research Bioresources (JCRB) Cell Bank or RIKEN BioResource Center by comparison with the database of JCRB Cell Bank or RIKEN BioResource Center (Table SI). The evaluation values (28) were calculated for all cell lines in this study. Ca9-22 cells (Fig. S1 and Table SI) do not include the MSK-922 cells of head and neck SCC origin (29).

Construction of plasmids. The transcriptional start site for PLOD2 was predicted using the DataBase of Transcriptional Start Sites (https://dbtss.hgc.jp). The symbols of minus (-) and plus (+) represent the upstream and downstream sides of the transcriptional start site, respectively. The human PLOD2 promoter (bp -1,498 to +170 , position from transcriptional start site +1 ; nucleotides of 1,668 bp) was amplified with PCR from genomic DNA of HSC-2 oral cancer cells using KOD Fx Neo DNA polymerase (Toyobo Life Science) and ligated into the pGL4.10 Luc2 vector (Promega Corporation), which encoded the Firefly luciferase reporter gene, at the KpnI-NheI site. Firefly luciferase reporter constructs containing the 5'-deleted PLOD2 promoter $(-1,187$ to $+170,-315$ to +170$)$ were isolated using a PCR-based method. The thermocycling conditions for the amplification of $P L O D 2$ promoter region were as follows: $94^{\circ} \mathrm{C}$ for $2 \mathrm{~min}$, two-step for 40 cycles $\left(98^{\circ} \mathrm{C}\right.$ for $10 \mathrm{sec}$ and $68^{\circ} \mathrm{C}$ for $60 \mathrm{sec}$ ), $68^{\circ} \mathrm{C}$ for $5 \mathrm{~min}$. The forward and reverse primer sequences for PLOD2 promoter region were as follows: p1498-Luc forward, 5'-TTGGGTACCACGAGTCTC ACAGCACAGAT-3'; p1187-Luc forward, 5'-TTGGGTACCAC 
TCAGAGGACTGATGCTGT-3'; p315-Luc forward, 5'-TTG GGTACCGAGTCTAAGGCTCTCTTGGCA-3'; p1498-Luc, p1187-Luc and p315-Luc reverse, 5'-CCTACTAGTCACG TCTGGACTGTTTGCTC-3'. Wild-type STAT3 was cloned from the cDNA of normal human dermal fibroblast cells (cat. no. C-12302; PromoCell GmbH) using KOD Fx Neo and ligated into the pcDNA 3.1(+) vector (Invitrogen; Thermo Fisher Scientific, Inc.) at the EcoRV-XhoI site, and referred to as WT-STAT3. The PCR conditions were performed as described above and the primers were as follows: STAT3 forward, 5'-GTGCAGATATCCAATGGCCCAATGGAAT CAGCTA-3' and reverse, 5'-ATATCTCGAGTCACATGG GGGAGGTAGCGC-3'. The constitutively active form of the STAT3 mutant (A661C, N663C; alanine residue at position 661 and asparagine residue at position 663 were substituted to cysteine residues) was generated using the QuikChange Site-Directed Mutagenesis Kit (Stratagene; Agilent Technologies, Inc.) following the manufacturer's protocol, and referred to as CA-STAT3. The primer (5'-AAGATCATGGATTGTACCTGT ATCCTGGTGTCTCC-3') for STAT3 mutagenesis was used. The nucleotide sequences of all constructs obtained with PCR were confirmed by Sanger DNA sequencing using ABI BigDye ${ }^{\mathrm{TM}}$ Terminator v3.1 Cycle Sequencing Kit and 3130 Genetic Analyzer (Applied Biosystem; Thermo Fisher Scientific, Inc.), according to the manufacturer's instructions.

Dual-luciferase reporter assay. Ca9-22 cells were cultured in $24-w e l l$ plates $(1 \times 10$ cells/well) for $24 \mathrm{~h}$. The following day, cells were co-transfected with the Firefly luciferase reporter plasmid containing a $P L O D 2$ promoter $(250 \mathrm{ng})$ and the control reporter vector expressing Renilla luciferase (pRL-TK, 25 ng; Promega Corporation) as an internal control for normalization of Firefly luciferase activity in comparison with Renilla luciferase activity. Transfections were performed using Lipofectamine ${ }^{\circledR} 3000$ reagent (Thermo Fisher Scientific, Inc.) according to the manufacturer's instructions. After $16 \mathrm{~h}$ of transfection, $0.5 \mathrm{ng} / \mathrm{ml}$ recombinant human IL-6 (cat. no. 206-IL-010; R\&D Systems, Inc.) was added to the medium, and cells were cultured at $37^{\circ} \mathrm{C}$ for $24 \mathrm{~h}$. Luciferase activity was measured with the Dual-Luciferase Reporter Assay System (Promega Corporation), according to the manufacturer's protocol. The luminescence was detected with a Centro XS3 LB 960 luminometer (Titertek-Berthold).

Cell invasion and migration analysis. PLOD2 small interfering (si)RNA (siPLOD2; ON-TARGETplus SMART pool; cat. no. L-004285-01-0005; Horizon Discovery, Ltd.) and Control siRNA (siControl; ON-TARGETplus Non-targeting Control pool; cat. no. D-001810-10-05; Horizon Discovery, Ltd.) was transfected into green fluorescence protein (GFP)-expressing oral cancer cell lines using Lipofectamine ${ }^{\circledR}$ RNAiMAX reagent (Thermo Fisher Scientific, Inc.) according to the manufacturer's protocol. After each siRNA ( $25 \mathrm{nM})$ transfection for $24 \mathrm{~h}$, siPLOD2 and siControl-transfected cells $\left(5 \times 10^{5}\right.$ cells/well in RPMI-1640 medium) were cultured at $37^{\circ} \mathrm{C}$ for $24 \mathrm{~h}$ into the upper chambers $(0.5 \% \mathrm{FBS})$ of the Transwell chambers (Corning, Inc.) coated with $0.1 \mathrm{mg} / \mathrm{ml}$ Matrigel $\left(37^{\circ} \mathrm{C}\right.$ for $2 \mathrm{~h}$; BD Biosciences) and allowed to invade toward the lower chambers that contained $10 \%$ FBS with or without IL-6. After $24 \mathrm{~h}$ of incubation, invaded GFP-expressing cells were detected with a fluorescence microscope (Olympus IX71; Olympus Corporation) using the $4 \mathrm{X}$ objective lens, and the number of cells was counted in the fields using cellSens standard software version 1.4 (Olympus Corporation). Cell migration with IL-6 treatment was detected with a wound healing assay using a 24 -well plate coated with COL-I (Corning, Inc.). When the confluence of GFP-expressing oral cancer cells reached $\sim 90-100 \%$ in RPMI-1640 medium with $10 \%$ FBS $(30,31)$, scratch wounds were created in each well. After scratching, the debris was removed and IL- 6 was added, the cell images were obtained after $0,6,12 \mathrm{~h}$ of incubation at $37^{\circ} \mathrm{C}$ and the migration of GFP-expressing cells were detected with an Olympus IX71 fluorescence microscope (Olympus Corporation) using the $2 \mathrm{X}$ objective lens. Migration was calculated as the relative percentage of scratch area to the area at $0 \mathrm{~h}$ (\% of wound closure) using cellSens standard software version 1.4 (Olympus Corporation).

Reverse transcription (RT)-semi-quantitative PCR. Total RNA was extracted from prepared cells using the RNeasy Plus Mini Kit (Qiagen, Inc.) according to the manufacturer's protocol. For analysis of mRNA, cDNA was synthesized from $1 \mu \mathrm{g}$ total RNA using ReverTra Ace ${ }^{\mathrm{TM}}$ (Toyobo Life Science) according to the manufacturer's protocol. cDNA was amplified by PCR with Z-Taq DNA Polymerase (Takara Bio, Inc.). The thermocycling conditions for PCR were as follows: $94^{\circ} \mathrm{C}$ for $150 \mathrm{sec}$, two-step for 35 cycles $\left(98^{\circ} \mathrm{C}\right.$ for $2 \mathrm{sec}$ and $68^{\circ} \mathrm{C}$ for $5 \mathrm{sec}$ ), $72^{\circ} \mathrm{C}$ for $5 \mathrm{~min}$. All the RT-PCR products obtained were analyzed by gel electrophoresis using $2 \%$ agarose gel (cat. no. 1613102; Bio-Rad Laboratories, Inc.) containing ethidium bromide (cat. no. 15585011; Invitrogen; Thermo Fisher Scientific, Inc.). $\beta$-actin was used as a positive control and for the normalization of RT-PCR products. PCR primer sets were previously described by Ueki et al (23). The PCR products were detected using a ChemiDoc ${ }^{\mathrm{TM}}$ Touch Imaging System (Bio-Rad Laboratories, Inc.) and band intensity was analyzed using Image Lab software version 5.2 (Bio-Rad Laboratories, Inc.).

Immunoblotting. The cell pellets were lysed with $100 \mathrm{ml}$ RIPA buffer [50 mM Tris/HCl (pH 7.5), $150 \mathrm{mM} \mathrm{NaCl}$, $0.5 \%$ sodium deoxycholate, $1 \%$ NP- $40,0.1 \%$ SDS] containing Complete Mini Protease Inhibitor (Roche Diagnostics) and with sonication for $30 \mathrm{sec}$. The supernatant was collected as soluble sample by centrifugation at $16,900 \mathrm{x}$ g for $30 \mathrm{~min}$ at $4^{\circ} \mathrm{C}$. The sample was diluted with 2 volumes of $2 \mathrm{X}$ Laemmli sample buffer (cat. no. 161-0737; Bio-Rad Laboratories, Inc.) and boiled at $98^{\circ} \mathrm{C}$ for $5 \mathrm{~min}$. Then, $20 \mathrm{ml}$ protein sample per lane was resolved via SDS-PAGE on a $10 \%$ gel. Separated proteins were then transferred to a nitrocellulose membrane and the membranes were blocked for $1 \mathrm{~h}$ at room temperature with 5\% skimmed milk (FUJIFILM Wako Pure Chemical Corporation) in TBS with Tween-20 (TBST; $50 \mathrm{mM}$ Tris- $\mathrm{HCl}$ $\mathrm{pH} 7.2,140 \mathrm{mM} \mathrm{NaCl}, 0.05 \%$ Tween-20). The following primary antibodies were diluted in TBST containing $5 \%$ skimmed milk and incubated overnight at $4^{\circ} \mathrm{C}$ : Rabbit anti-PLOD2 (1:1,000; cat. no. 21214-1-AP; ProteinTech Group, Inc.), rabbit anti-integrin $\beta 1$ (1:1,000; cat. no. 9699; Cell Signaling Technology, Inc.) and mouse anti-human $\beta$-actin antibody (1:5,000; cat. no. MAB1501; EMD Millipore). 
Membranes were then incubated for $1 \mathrm{~h}$ at room temperature with horseradish peroxidase-conjugated anti-mouse (1:10,000; cat. no. 62-6520; Thermo Fisher Scientific, Inc.) or anti-rabbit IgG secondary antibodies (1:10,000; cat. no. 65-6120; Thermo Fisher Scientific, Inc.). Positive signals were enhanced with a chemiluminescence system (Supersignal West Pico substrate; Thermo Fisher Scientific, Inc.) and visualized using the ChemiDoc $^{\mathrm{TM}}$ Touch Imaging System.

Immunocytochemistry. Oral cancer cell lines (HSC-2, HSC-3, Ca9-22) treated with or without IL-6 were fixed for $30 \mathrm{~min}$ at room temperature with $4 \%$ paraformaldehyde in phosphate-buffered saline (PBS) and permeabilized with $0.1 \%$ Triton X-100 (cat. no. T8787; Sigma-Aldrich; Merck KGaA) in PBS for $3 \mathrm{~min}$ at room temperature. After blocking with 3\% BSA (cat. no. A7030; Sigma-Aldrich; Merck KGaA) in TBST, these cells were incubated with primary antibodies in $3 \% \mathrm{BSA} / \mathrm{TBST}$ overnight at $4^{\circ} \mathrm{C}$. PLOD2 expression was detected by staining with a rabbit anti-PLOD2 primary antibody (1:500; cat. no. 21214-1-AP; ProteinTech Group, Inc.), and integrin $\beta 1$ expression was detected by staining with mouse anti-integrin $\beta 1$ primary antibody (1:500; cat. no. ab3167; Abcam). Cells were washed three times with $3 \% \mathrm{BSA} / \mathrm{TBST}$ and incubated for $1 \mathrm{~h}$ at room temperature with secondary antibodies in $3 \% \mathrm{BSA} / \mathrm{PBST}$, and then washed three times with PBS. The secondary antibodies used were Cy3-labeled goat anti-rabbit $(\mathrm{H}+\mathrm{L})$ antibody (1:500; cat. no. 111-167-003; Jackson ImmunoResearch Laboratories, Inc.) and Alexa Fluor 555-labeled goat anti-mouse IgG $(\mathrm{H}+\mathrm{L})$ antibody (1:500; cat. no. A28180; Thermo Fisher Scientific, Inc.). Cell nuclei were counterstained with 4',6-diamidino-2-phenylindole (1:1,000; Hoechst 33342 dye: Molecular Probes; Thermo Fisher Scientific, Inc.) for $1 \mathrm{~min}$ at room temperature. CellLight $^{\circledR}$ ER-GFP BacMam 2.0 (Thermo Fisher Scientific, Inc.) was used as an endoplasmic reticulum (ER) marker. The fluorescence signals were detected with an Olympus IX71 fluorescence microscope and analyzed using cellSens Standard software version 1.4 (Olympus Corporation).

Immunohistochemistry and immunofluorescence staining. The use of patient-derived tissues was approved by the ethics committees of Niigata University Graduate School of Medical and Dental Sciences (approval no. 2019-0173; Niigata, Japan). The cohort comprised 15 patients $>20$ years of age with histopathologically-confirmed head and neck cancer, including oral cancer, who had been surgically treated at the Niigata University Hospital (Niigata, Japan) between January 2015 and June 2019. Consent was not obtained from each patient, however the patients were notified of the details of the study by opt-out consent and had the right to refuse participation in the study. Human oral and laryngeal carcinoma tissues were fixed in $10 \%$ buffered formalin solution for $24 \mathrm{~h}$ at room temperature. Paraffin-embedded sections $(5 \mathrm{~mm}$ ) were deparaffinized, and antigen retrieval was performed by autoclaving (Pascal S2800; Dako; Agilent Technologies, Inc.) in sodium citrate buffer. Endogenous peroxidase activity was quenched by incubation in $0.3 \%$ hydrogen peroxide (Kanto Chemical Co., Inc.) for $20 \mathrm{~min}$ at room temperature. Sections were blocked with $1 \%$ BSA in PBS for 30 min at room temperature, and then incubated with rabbit anti-IL-6 (1:200; cat. no. 21865-1-AP;
ProteinTech Group, Inc.) and mouse anti-CD163 (1:500; cat. no. NCL-L-CD163; Leica Biosystems) primary antibodies overnight at $4^{\circ} \mathrm{C}$. After the sections were washed with PBS, they were subjected to the diaminobenzidine detection system (EnVision+ Kit/horseradish peroxidase; Dako; Agilent Technologies, Inc.) according to the manufacturer's protocol. For fluorescence immunostaining of tissues, primary antibodies were used against IL-6 and CD163, and tissues were incubated for $1 \mathrm{~h}$ at room temperature with $\mathrm{Cy} 3$-conjugated goat anti-rabbit $\operatorname{IgG}(\mathrm{H}+\mathrm{L})$ secondary antibody (1:500; cat. no. 111-167-003; Jackson ImmunoResearch Laboratories, Inc.) and Alexa Fluor 488-conjugated goat anti-mouse IgG $(\mathrm{H}+\mathrm{L})$ secondary antibody (1:500; cat. no. A-11017; Thermo Fisher Scientific, Inc.). The nuclei were stained with Hoechst 33258 $(1: 1,000)$ for $1 \mathrm{~min}$ at room temperature. The images were detected with an Olympus IX71 fluorescence microscope (Olympus Corporation) using the 20X objective lens and a sCMOS camera (Zyla 4.2 Plus; Andor Technology, Ltd.).

Statistical analysis. Data are shown as the mean \pm standard deviation from three independent experiments. Comparisons between two groups were carried out using a paired Student's t-test (Microsoft Excel version 2102; Microsoft Corporation). For multiple comparisons, one-way analysis of variance followed by Tukey's post hoc test was performed using Excel statistical software $(32,33)$ (BellCurve for Excel version 3.21, http://bellcurve.jp/; Social Survey Research Information Co., Ltd.). $\mathrm{P}<0.05$ was considered to indicate a statistically significant difference.

\section{Results}

PLOD2 induction by IL-6 in oral cancer cells. By searching the genomic sequence of human PLOD2, the STAT3 binding motif was predicted within the promoter region (Fig. 1A). Thus, the IL-6-STAT3 pathway may be involved in oral cancer progression (34). According to this hypothesis, it was next examined whether PLOD2 expression was altered in the presence of IL-6. As shown in Fig. 1B, the mRNA levels of PLOD2 in oral SCC (HSC-2, HSC-3 and Ca9-22) cells were notably elevated in response to IL-6. On the other hand, no marked changes in PLOD1 or PLOD3 mRNA levels was observed following treatment with IL-6. To examine IL-6-induced PLOD2 expression in detail, a luciferase reporter assay was performed in which luciferase was driven by the human PLOD2 promoter based on 1,498 bp that includes three intrinsic STAT3 binding sites (top) or deletion promoters based on 1,187 bp (middle) and $315 \mathrm{bp}$ (bottom) that lack one and all STAT3 binding elements (Fig. 1A). Using this approach, it was found that the expression of PLOD2 promoter-reporter constructs, p1498-Luc and p1187-Luc, that contain the indicated STAT3 binding sites (three for p1498-Luc and two for p1187-Luc) were both upregulated by IL-6 stimulation, but their expression levels were different (Fig. 1C). However, the PLOD2 promoter construct, p315-Luc, which lacks all STAT3-binding cis-elements showed almost no response to IL-6. Furthermore, forced expression of an expression vector carrying CA-STAT3, but not WT-STAT3, significantly induced the expression of the luciferase reporter gene from co-transfected reporter constructs (Fig. 1D). However, even 
A

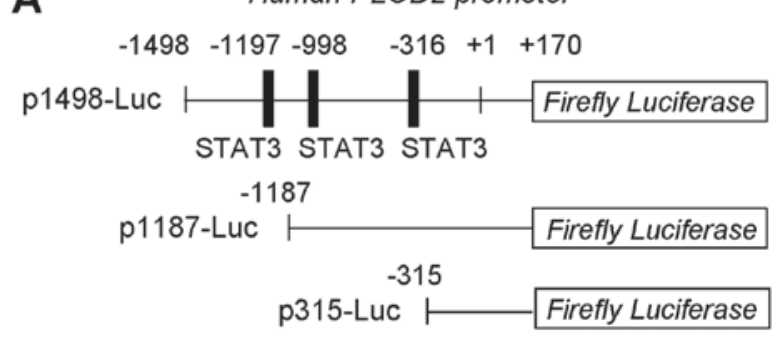

Putative STAT3

\begin{tabular}{|c|c|}
\hline binding site & $5^{\prime}$ to $3^{\prime}$ \\
\hline-316 to -326 & ATTACTTGGAAA \\
\hline-998 to -1008 & GITCTGAGAAG \\
\hline-1197 to -1207 & CIGETAGGAAT \\
\hline $\begin{array}{c}\text { Consensus } \\
\text { sequence }\end{array}$ & CTTC \\
\hline
\end{tabular}

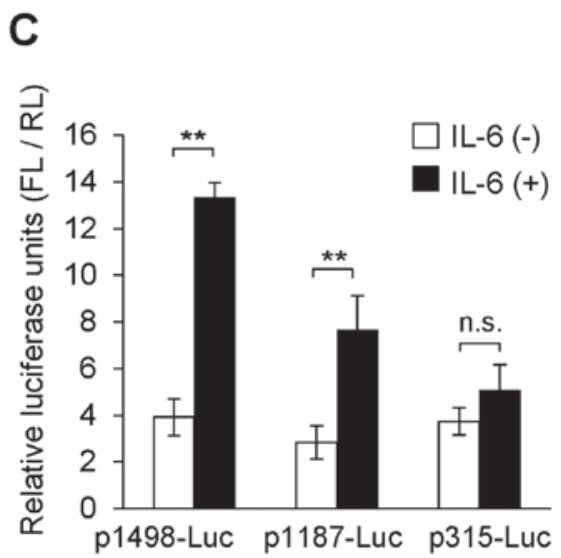

E

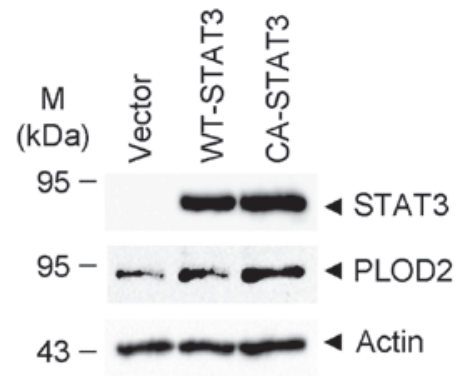

Figure 1. PLOD2 induction of oral cancer cells by IL-6 stimulation. (A) Construction of the human PLOD2 promoter-reporter system and the deletion mutants of STAT3-binding elements in the PLOD2 promoter are shown. Sequences and positions of putative STAT3 binding sites on the PLOD2 promoter are shown. (B) Oral cancer cells (HSC-2, HSC-3 and Ca9-22) were stimulated with IL-6 at the indicated concentrations for 48 h, and the mRNA levels of PLOD family members were detected via reverse transcription-semi-quantitative PCR. (C) Ca9-22 cells were co-transfected with the PLOD2 promoter-reporter plasmid (p1498-Luc, p1187-Luc and p315-Luc) and the RL vector as an internal control. Cells were treated with or without IL-6 (0.5 ng/ml) for 24 h, and the expression levels of FL and RL were measured with a luminometer. Each experiment was performed in triplicate and normalized to $\mathrm{RL}$ expression ( $=3$ ). ${ }^{* *} \mathrm{P}<0.01$. (D) Ca9-22 cells were co-transfected with the reporter plasmid and the RL vector. Then, $24 \mathrm{~h}$ after induction of reporter genes, cells were further transfected with WT-STAT3 or CA-STAT3. FL activity was normalized to RL expression as an internal control. Data are presented as the mean \pm SD from three independent experiments $(\mathrm{n}=3)$. ${ }^{* *} \mathrm{P}<0.01$. ANOVA followed by a Tukey's test. (E) Plasmid with WT-STAT3 or CA-STAT3 was transiently transfected into Ca9-22 cells. The expression of PLOD2 was detected via immunoblotting. PLOD2, procollagen-lysine 2-oxoglutarate 5-dioxygenase 2; IL-, interleukin; STAT3, signal transducer and activator of transcription 3; n.s., not significant; WT, wild-type; CA, constitutively activate; FL, Firefly luciferase; RL, Renilla luciferase.

in the presence of CA-STAT3, the expression levels of luciferase following transfection of p315-Luc were comparable to levels in the presence of IL-6 (Fig. 1C). As shown in Fig. 1D, the overexpression of CA-STAT3, but not WT-STAT3, notably upregulated endogenous PLOD2 in the transfected cells that were not treated with IL-6 (Fig. 1E). These results indicated that PLOD2 expression is positively regulated by the IL-6-mediated canonical pathway, IL-6-STAT3, in oral cancer cells.

Integrin $\beta 1$ activation via the IL-6/STAT3/PLOD2 signaling axis. In the cell invasion assay using a Matrigel-coated Transwell chamber, IL-6 stimulation of oral SCC cells increased their invasiveness. Conversely, transfection with siPLOD2 significantly suppressed cell invasion and migration, even in the presence of IL-6 (Figs. 2A and S2A and B). We previously reported that PLOD2 increases cancer metastasis through stabilization of integrin $\beta 1$ protein on the cancer cell surface via PLOD2-catalyzed lysyl hydroxylation (23). Therefore, the present study assessed whether the integrin $\beta 1$ state is affected by PLOD2 itself and IL- 6 treatment. As shown in Fig. 2B, the protein levels of integrin $\beta 1$ in all three cell lines were efficiently downregulated following transfection with siPLOD2. In addition, the integrin $\beta 1$ content in individual cells was elevated with the increase in PLOD2 levels in response to IL-6 stimulation (Fig. 2C). However, no significant 
A

HSC-2
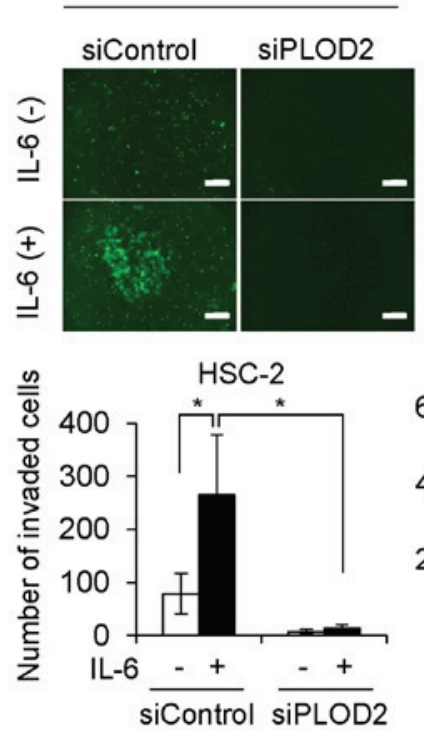

HSC-3

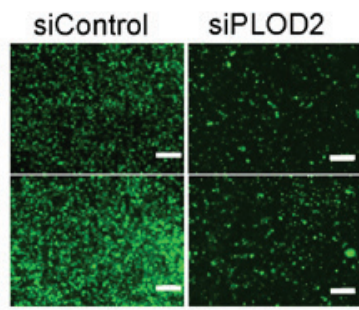

HSC-3

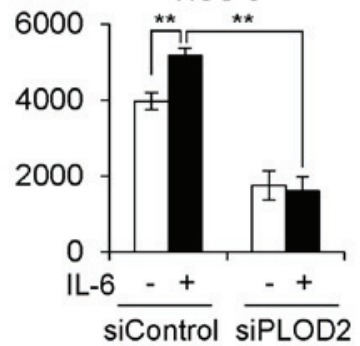

Ca9-22

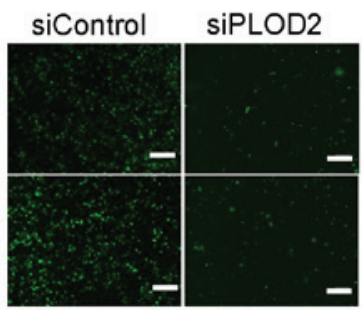

Ca9-22

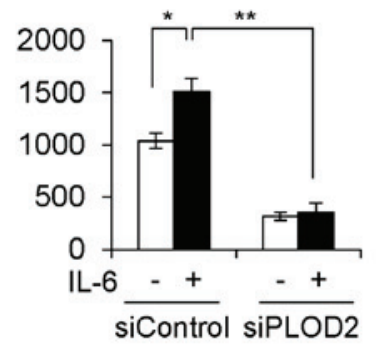

B

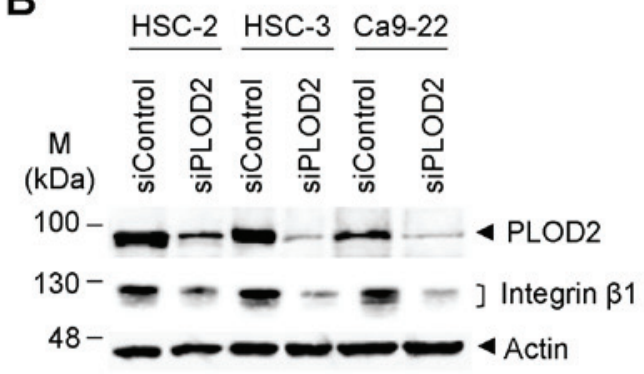

C

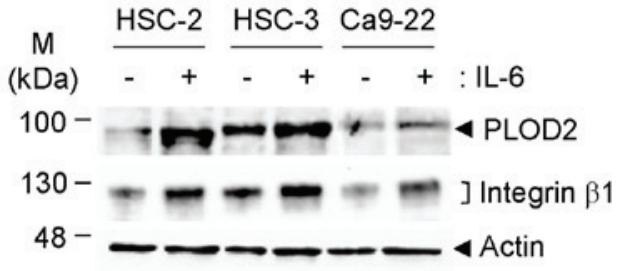

D

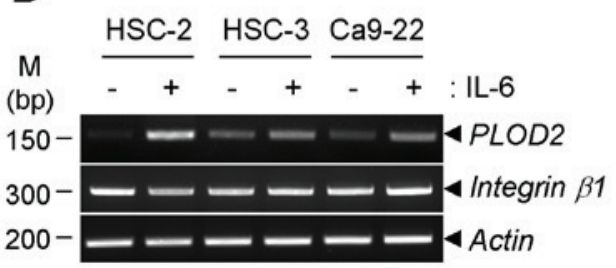

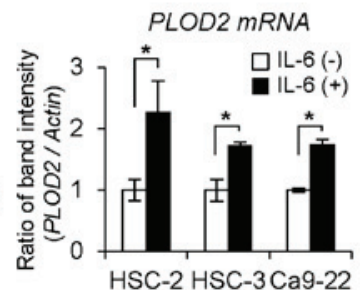

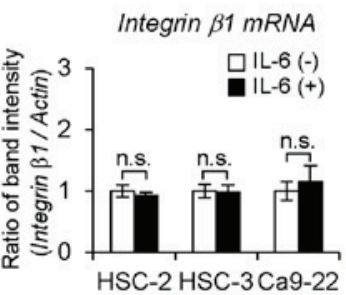

Figure 2. Invasion of oral cancer cells in response to IL-6. (A) siPLOD2 and siControl-transfected oral cancer cells (HSC-2, HSC-3 and Ca9-22, green fluorescence protein-positive cells) were seeded into the upper chamber of a Matrigel-coated Transwell plate. The cells were treated with or without IL- 6 for 24 h, and then cells that had migrated to the lower chamber were observed with fluorescence microscopy (upper panels). The number of migrated cells was calculated from the fluorescence image (lower panels) $(n=3)$. ${ }^{*} \mathrm{P}<0.05,{ }^{* *} \mathrm{P}<0.01$. ANOVA followed by a Tukey's test. Data are presented as the mean $\pm \mathrm{SD}$ from three independent experiments. Scale bar, $200 \mu \mathrm{m}$. (B) Expression levels of integrin $\beta 1$ in siPLOD2-transfected cells were detected with immunoblotting. (C) Oral cancer cells were stimulated with IL-6 $(0.5 \mathrm{ng} / \mathrm{ml})$ for $48 \mathrm{~h}$, and the expression levels of PLOD2 and integrin $\beta 1$ were detected via immunoblotting. (D) Transcript levels of $P L O D 2$ and integrin $\beta 1$ with or without IL- 6 were determined via reverse transcription-semi-quantitative PCR (left panels). The band intensity of PCR products was semi-quantified by densitometric analysis and normalized to $\beta$-actin band intensity (right panels). Data are presented as the mean \pm SD from three independent experiments $(n=3)$. ${ }^{*} \mathrm{P}<0.05$. PLOD2, procollagen-lysine 2-oxoglutarate 5-dioxygenase 2; IL-, interleukin; siRNA/si, small interfering; n.s., not significant.

alteration in integrin $\beta 1 \mathrm{mRNA}$ expression with or without IL-6 stimulation was detected in oral SCC cells (Fig. 2D). Therefore, IL-6 in these conditions did not affect induction of integrin $\beta 1 \mathrm{mRNA}$. The results suggested that integrin $\beta 1$ protein is regulated by PLOD2 in tumor cells.

Intracellular localization of PLOD2 and integrin $\beta 1$ by IL- 6 . Although the ER localization of PLOD2 was not changed in the absence of IL- 6 compared with the presence of IL-6 (Fig. 3A), integrin $\beta 1$ was actively recruited to the plasma membrane in response to IL-6. It was further confirmed that the IL-6-enhanced plasma membrane localization of integrin $\beta 1$ was notably downregulated by transfection with siPLOD2 (Fig. 3B). Therefore, these results indicated that the localization of integrin $\beta 1$ was influenced by the ER localization of PLOD2.

To examine whether IL- 6 induces a functional, mature form of integrin $\beta 1$ on the plasma membrane, western blotting was performed to detect the mature form of the protein, which has a molecular weight of $125 \mathrm{kDa}$. IL- 6 induced the upregulation of PLOD2, which was followed by an increase in the mature form of integrin $\beta 1$ (upper band) in cells expressing 


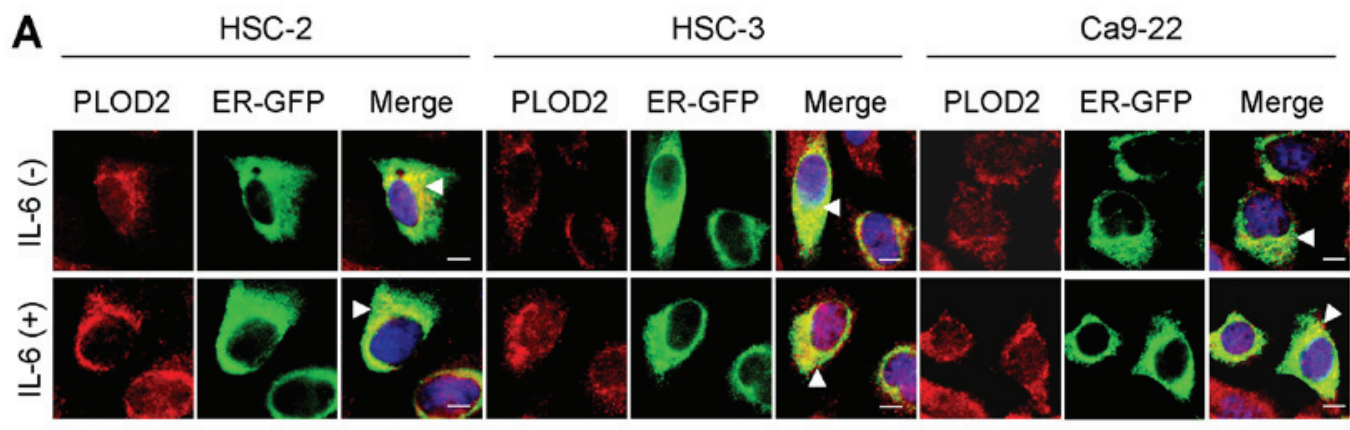

B
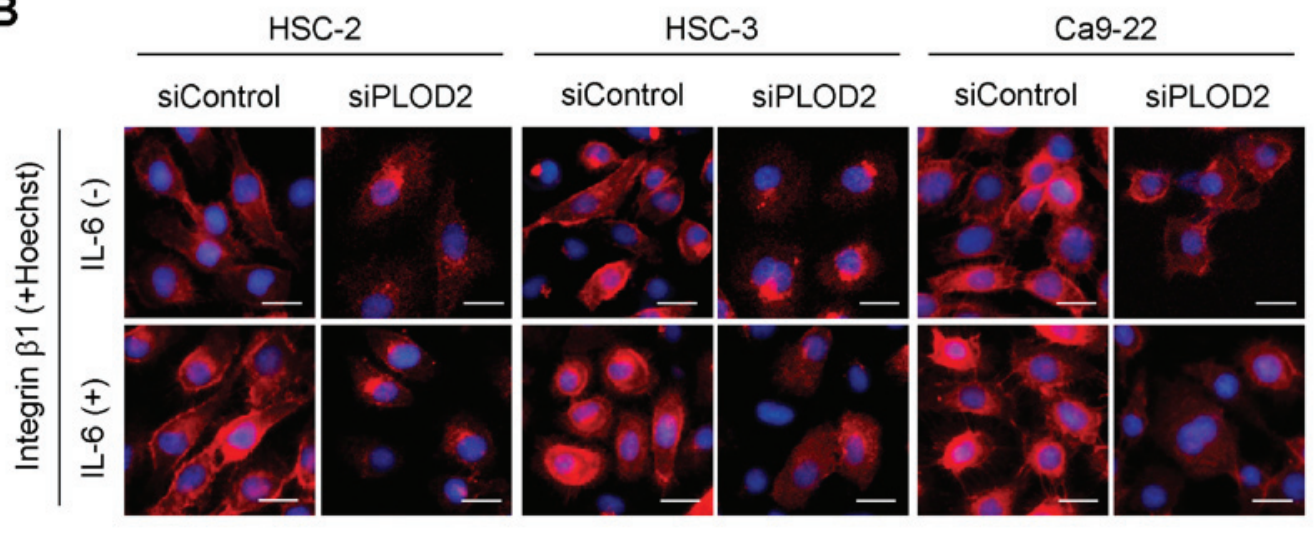

C

D
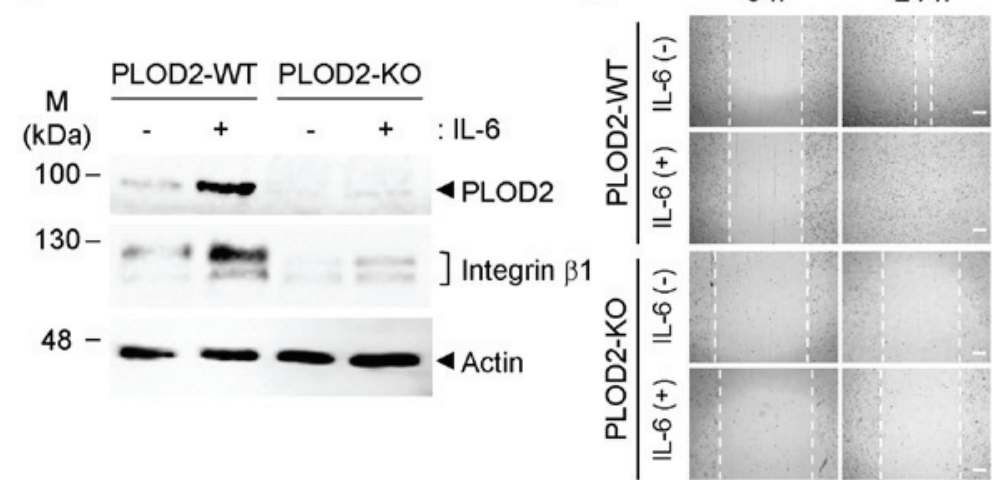

$\rightarrow$ PLOD2-WT

$\rightarrow-$ PLOD2-WT + IL-6

$\rightarrow-\mathrm{PLOD} 2-\mathrm{KO}$

$\rightarrow$ PLOD2-KO + IL-6

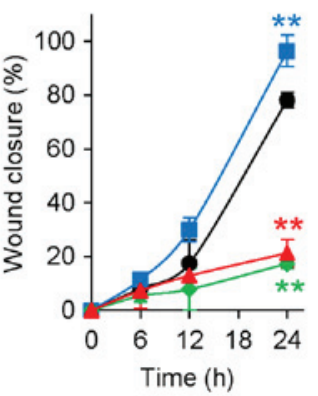

Figure 3. PLOD2-dependent intracellular localization of integrin $\beta 1$. (A) Intracellular localization of PLOD2 (red) with or without IL-6 treatment was observed with immunostaining. Localization of PLOD2 was merged with the ER marker, ER-GFP (arrowhead). Nuclei were stained with Hoechst 33258. Scale bar, $10 \mu \mathrm{m}$. (B) Localization and expression of integrin $\beta 1$ (red) were observed with immunocytochemistry staining $48 \mathrm{~h}$ after IL-6 and siPLOD2 stimulation. Nuclei (blue) were stained with Hoechst 33258. Scale bar, $20 \mu \mathrm{m}$. (C) Expression levels of PLOD2 and integrin $\beta 1$ in PLOD2-KO HSC-2 cells were detected with immunoblot analysis. (D) Cell migration of PLOD2-KO cell lines with or without IL-6 treatment ( $0.5 \mathrm{ng} / \mathrm{ml})$ was detected with a wound healing assay using a 24-well plate. Images were taken 0 and $24 \mathrm{~h}$ after wound formation (scale bar, $400 \mu \mathrm{m}$ ), and the wound width was estimated using microscopic images. Data are presented as the mean $\pm \mathrm{SD}(\mathrm{n}=3)$. ANOVA followed by a Tukey's test. ${ }^{* *} \mathrm{P}<0.01$ vs. PLOD2-WT without IL- 6 treatment group. PLOD2, procollagen-lysine 2-oxoglutarate 5-dioxygenase 2; IL-, interleukin; siRNA/si, small interfering; GFP, green fluorescence protein; ER, endoplasmic reticulum; KO, knockout; WT, wild-type.

PLOD2-WT (Fig. 3C). On the other hand, the mature form of integrin $\beta 1$ in PLOD2-KO cell lines was expressed at lower levels than that of integrin $\beta 1$ in PLOD2-WT. The lower generation of the mature form of integrin $\beta 1$ in PLOD2-KO resulted in a reduction in cell migration regardless of IL-6 treatment (Fig. 3D). Taken together, these results indicated that the PLOD2-integrin $\beta 1$ axis in which PLOD2 induces maturation and membrane localization of integrin $\beta 1$ increases cell migration and invasion in oral cancer cells and is triggered by extracellular IL-6.

Endogenous IL-6 expression in oral and neck SCC tissues. To identify the main source of IL-6 in the oral TME, the in situ distribution of IL-6 expression was investigated in tissue sections prepared from surgically resected specimens of oral and laryngeal SCCs. IL-6 was highly expressed in the tumor stroma and co-localized with CD163-positive M2 macrophages (Fig. 4A, arrows). The co-localization of IL-6 and CD163 was confirmed with double-immunofluorescence staining (Fig. 4B). The staining patterns of IL-6 were highly similar to those of CD163 in the tumor stromal areas in all oral SCC tissues examined. Overall, it was observed that CD163-positive M2 macrophages in the oral TME likely secrete IL-6, which activates oral cancer cells and increases their migratory and invasive capabilities via the PLOD2-integrin $\beta 1$ signaling axis. 
A

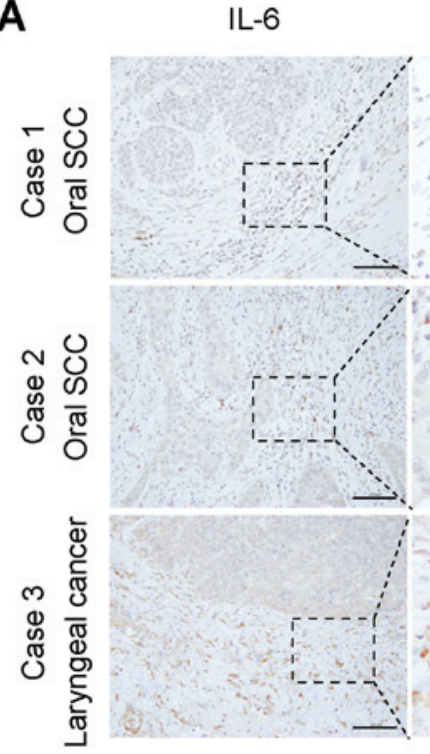

IL-6

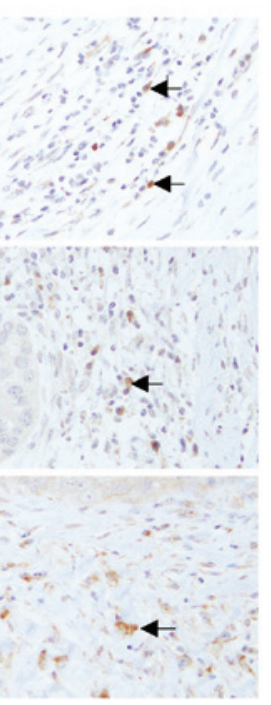

CD163

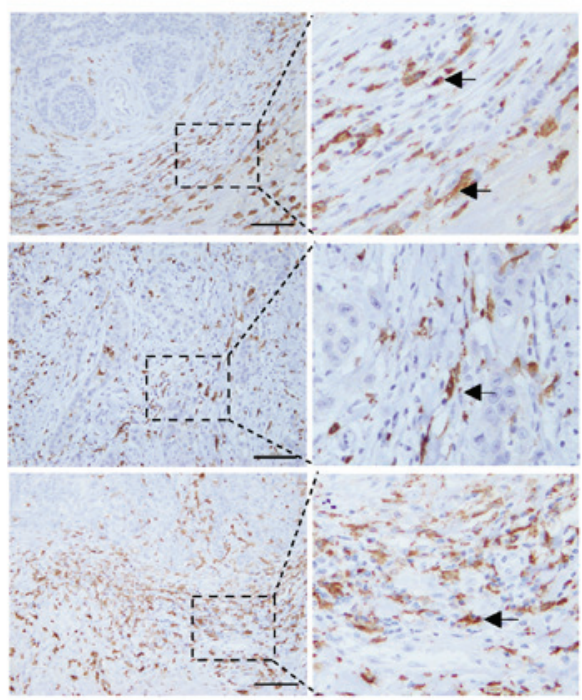

B

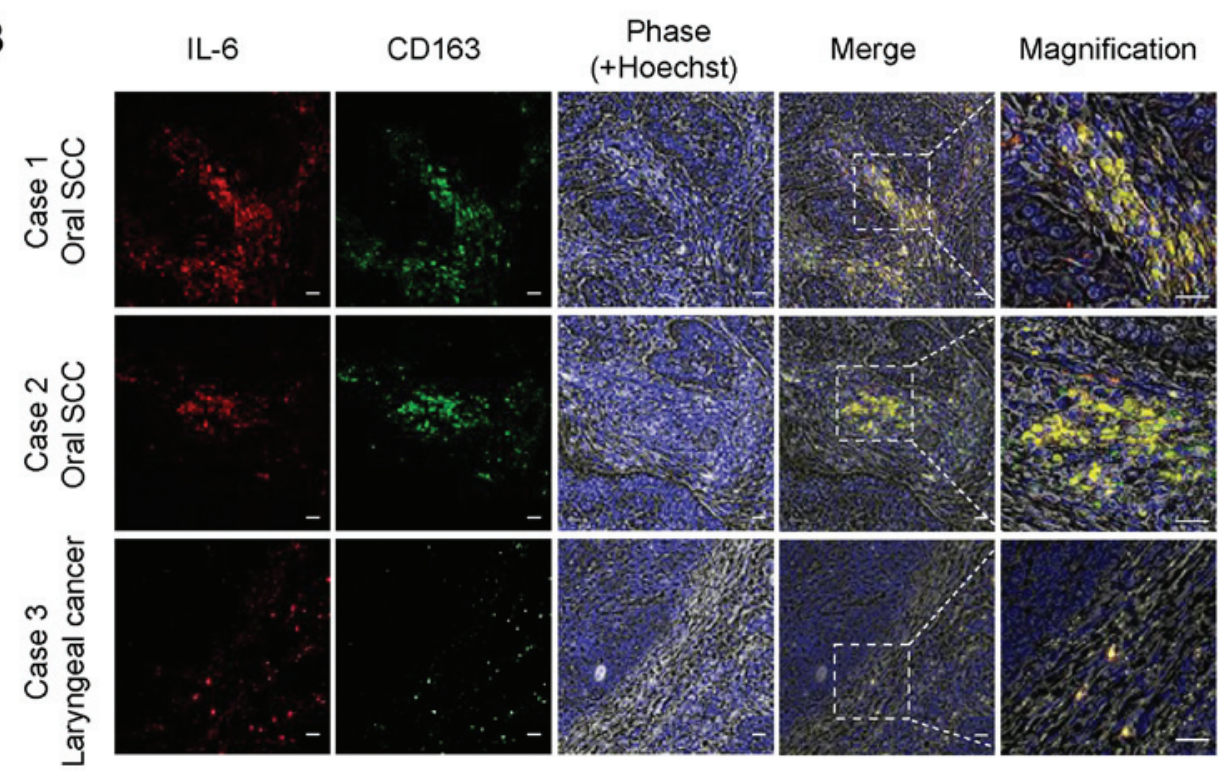

Figure 4. Expression of IL-6 by M2 macrophages in patient tumor tissues. (A) Immunohistochemistry staining for IL-6 and CD163 is shown in oral SCC (cases 1,2) and laryngeal cancer (case 3). A high-power tumor stroma field (dashed line box) is shown on the right (magnification, x20). Co-localization in cells is indicated by arrowheads. Scale bar, $200 \mu \mathrm{m}$. (B) IL-6 (red) and CD163 (green) were observed with immunofluorescence analysis. Magnification (magnification, $\mathrm{x} 2.5$ ) of the merged area (dashed line box) is shown on the right. Scale bar, $20 \mu \mathrm{m}$. IL-, interleukin; SCC, squamous cell carcinoma.

\section{Discussion}

Accumulating evidence indicates that PLOD2 is highly expressed in cancer cells, and collagen molecules that mainly comprise the tumor ECM have been identified as its major substrate $(13,14,35)$. Therefore, at present, research on PLOD2 in cancer biology has focused on the regulation of the tumor ECM via the interaction of PLOD2 with extracellular collagens in the TME. We recently reported that integrin $\beta 1$ is an important substrate for PLOD2 in cancer cells that plays a role in cancer progression (23). This previous study demonstrated that PLOD2 directly catalyzes the hydroxylation of integrin $\beta 1$, which leads to increased stability, plasma membrane recruitment and conversion into the mature, active form of integrin $\beta 1$. Mature integrin $\beta 1$ readily interacts with tumor ECM collagens, resulting in accelerated cancer invasion and metastasis of oral SCC cells (23). Thus, we considered that
PLOD2 facilitates cancer metastasis via two functions: Effects on the tumor ECM in the tumor stroma and on integrin $\beta 1$ in cancer cells.

PLOD2 plays an unusual role in inducing metastasis of cancer cells. In the present study, it was hypothesized how PLOD2 expression is regulated in cancer cells. PLOD2 expression in breast cancer cells is induced by hypoxic conditions or co-cultivation with adipocytes $(19,35)$. Based on murine Plod2 promoter analysis, HIF-1 $\alpha$ and STAT3 transcription factors may play an important role in the induction of PLOD2 expression (14). IL-6-mediated activation of STAT3 is required for the induction of PLOD2 in oral cancer cells (23). Thus, the IL-6-STAT3 and hypoxia-HIF-1 $\alpha$ pathways may work together to induce PLOD2 expression. We are investigating this idea further.

In patients with oral SCC, IL- 6 is enriched in the oral TME and works to activate cancer cells. Thus, IL-6 may become 
a potential biomarker to diagnose tumor progression (24-27). Because the IL-6-STAT3 signaling axis in cancer cells plays a significant part in cancer progression (34), targeting IL-6 itself or the main source of IL-6 may be meaningful to prevent oral cancer progression. Based on this idea, we revealed overlap of IL-6 and CD163-positive macrophages in the stroma area of oral SCC tissues. CD163-positive macrophages may be TAMs with the M2 phenotype, which are closely correlated with poor prognosis in not only oral cancer, but also a variety of other cancer types, such as glioma, breast cancer, colorectal cancer and melanoma $(36,37)$. The number of TAMs increases in cases with higher grades of oral SCC (38). In particular, the M2 phenotype of TAMs is tightly associated with the induction of several cancer events, such as angiogenesis, growth, invasion and metastasis, through active production and secretion of numerous cytokines and chemokines, including IL-6. These findings combined with the current results suggest that IL-6 is actively produced and secreted from TAMs with the M2 phenotype that are present in the stroma area of oral SCC. IL-6 may promote cancer invasion and metastasis by enhancing the PLOD2-integrin $\beta 1$ signaling pathway in oral SCC cells.

We previously reported that PLOD2 signaling in oral SCC cells is involved in collective-cell migration, which occurs regardless of the expression state of E-cadherin or Snail (23). PLOD2 may also promote EMT in glioma (25). Because of the multiple mechanisms involved in cellular migration and invasion due to tumor heterogeneity and cancer cell plasticity (39), PLOD2 may have other substrates in addition to collagens and integrin $\beta 1$. Further studies of PLOD2 that include comprehensive identification of PLOD2 substrates and binding partners will increase our understanding of cancer invasion and metastasis, as well as the signaling crosstalk between the tumor and tumor stroma.

\section{Acknowledgements}

We would like to thank Dr Homma and Dr Kawasaki (Niigata Cancer Center Hospital, Niigata, Japan) and Dr Ueki (Department of Otolaryngology, Head and Neck Surgery, Niigata University, Niigata, Japan) for providing the pathological findings for the grade origin and local invasiveness of oral squamous cell carcinoma.

\section{Funding}

This work was supported by Niigata Medical Association (Yujin Memorial Grant for 2020).

\section{Availability of data and materials}

The datasets used and/or analyzed in the present study are available from the corresponding author on reasonable request.

\section{Authors' contributions}

KS designed the outline of the study. KS, AM, IWS and YY conducted the experiments and contributed to data interpretation and manuscript preparation. KS and AM confirmed the authenticity of all the raw data. KS wrote the manuscript. MS and EK supervised the study and contributed to data interpretation and manuscript preparation/revision. All authors read and approved the final version of this manuscript.

\section{Ethics approval and consent to participate}

The use of patient-derived tissues was approved by the ethics committees of Niigata University Graduate School of Medical and Dental Sciences (approval no. 2019-0173). Consent was not obtained from each patient, however the patients were notified of the details of the study by opt-out consent and have the right to refuse participation in the study. This is a method widely used in Japan. All study procedures adhered to the principles of the Declaration of Helsinki.

\section{Patient consent for publication}

Not applicable.

\section{Competing interests}

The authors declare that they have no competing interests.

\section{References}

1. Bettendorf $\mathrm{O}$, Piffkò $\mathrm{J}$ and Bànkfalvi A: Prognostic and predictive factors in oral squamous cell cancer: Important tools for planning individual therapy? Oral Oncol 40: 110-119, 2004.

2. Fong D, Spizzo G, Gostner JM, Gastl G, Moser P, Krammel C, Gerhard S, Rasse M and Laimer K: TROP2: A novel prognostic marker in squamous cell carcinoma of the oral cavity. Mod Pathol 21: 186-191, 2008.

3. Markolovic S, Wilkins SE and Schofield CJ: Protein hydroxylation Catalyzed by 2-oxoglutarate-dependent oxygenases. J Biol Chem 290: 20712-20722, 2015.

4. Ploumakis A and Coleman ML: OH, the places you'll go! hydroxylation, gene expression, and cancer. Mol Cell 58: 729-741, 2015.

5. Klose RJ, Kallin EM and Zhang Y: JmjC-domain-containing proteins and histone demethylation. Nat Rev Genet 7: 715-727, 2006.

6. Gilkes DM, Chaturvedi P, Bajpai S, Wong CC, Wei H, Pitcairn S, Hubbi ME, Wirtz D and Semenza GL: Collagen prolyl hydroxylases are essential for breast cancer metastasis. Cancer Res 73: 3285-3296, 2013.

7. Myllyharju J: Prolyl 4-hydroxylases, the key enzymes of collagen biosynthesis. Matrix Biol 22: 15-24, 2003.

8. Lee KA, Lynd JD, O'Reilly S, Kiupel M, McCormick JJ and LaPres JJ: The biphasic role of the hypoxia-inducible factor prolyl-4-hydroxylase, PHD2, in modulating tumor-forming potential. Mol Cancer Res 6: 829-842, 2008.

9. Epstein AC, Gleadle JM, McNeill LA, Hewitson KS, O'Rourke J, Mole DR, Mukherji M, Metzen E, Wilson MI, Dhanda A, et al: C. elegans EGL-9 and mammalian homologs define a family of dioxygenases that regulate HIF by prolyl hydroxylation. Cell 107: 43-54, 2001.

10. Bruick RK and McKnight SL: A conserved family of prolyl-4-hydroxylases that modify HIF. Science 294: 1337-1340, 2001.

11. Højfeldt JW, Agger K and Helin K: Histone lysine demethylases as targets for anticancer therapy. Nat Rev Drug Discov 12: 917-930, 2013.

12. Black JC, Manning AL, Van Rechem C, Kim J, Ladd B, Cho J, Pineda CM, Murphy N, Daniels DL, Montagna C, et al: KDM4A lysine demethylase induces site-specific copy gain and rereplication of regions amplified in tumors. Cell 154: 541-555, 2013.

13. Takaluoma K, Lantto J and Myllyharju J: Lysyl hydroxylase 2 is a specific telopeptide hydroxylase, while all three isoenzymes hydroxylate collagenous sequences. Matrix Biol 26: 396-403, 2007.

14. Chen Y, Terajima M, Yang Y, Sun L, Ahn YH, Pankova D, Puperi DS, Watanabe T, Kim MP, Blackmon SH, et al: Lysyl hydroxylase 2 induces a collagen cross-link switch in tumor stroma. J Clin Invest 125: 1147-1162, 2015. 
15. Gilkes DM, Bajpai S, Wong CC, Chaturvedi P, Hubbi ME, Wirtz D and Semenza GL: Procollagen lysyl hydroxylase 2 is essential for hypoxia-induced breast cancer metastasis. Mol Cancer Res 11: 456-466, 2013.

16. Gilkes DM, Bajpai S, Chaturvedi P, Wirtz D and Semenza GL: Hypoxia-inducible factor 1 (HIF-1) promotes extracellular matrix remodeling under hypoxic conditions by inducing P4HA1, P4HA2, and PLOD2 expression in fibroblasts. J Biol Chem 288: 10819-10829, 2013.

17. Gjaltema RAF, de Rond S, Rots MG and Bank RA: Procollagen lysyl hydroxylase 2 expression is regulated by an alternative downstream transforming growth factor b-1 activation mechanism. J Biol Chem 290: 28465-28476, 2015.

18. Noda T, Yamamoto H, Takemasa I, Yamada D, Uemura M, Wada H, Kobayashi S, Marubashi S, Eguchi H, Tanemura M, et al: PLOD2 induced under hypoxia is a novel prognostic factor for hepatocellular carcinoma after curative resection. Liver Int 32: 110-118, 2012.

19. He JY, Wei XH, Li SJ, Liu Y, Hu HL, Li ZZ, Kuang XH, Wang L, Shi X, Yuan ST, et al: Adipocyte-derived IL-6 and leptin promote breast cancer metastasis via upregulation of lysyl hydroxylase-2 expression. Cell Commun Signal 16: 100-118, 2018.

20. Levental KR, Yu H, Kass L, Lakins JN, Egeblad M, Erler JT, Fong SF, Csiszar K, Giaccia A, Weninger W, et al: Matrix crosslinking forces tumor progression by enhancing integrin signaling. Cell 139: 891-906, 2009.

21. Li Z, Liu FY and Kirkwood KL: The p38/MKP-1 signaling axis in oral cancer: Impact of tumor-associated macrophages. Ora Oncol 103: 104591-104599, 2020.

22. Solinas G, Germano G, Mantovani A and Allavena P Tumor-associated macrophages (TAM) as major players of the cancer-related inflammation. J Leukoc Biol 86: 1065-1073, 2009.

23. Ueki Y, Saito K, Iioka H, Sakamoto I, Kanda Y, Sakaguchi M, Horii A and Kondo E: PLOD2 Is Essential to functional activation of integrin $\beta 1$ for invasion/metastasis in head and neck squamous cell carcinomas. iScience 23: 100850, 2020.

24. St John MA, Li Y, Zhou X, Denny P, Ho CM, Montemagno C, Shi W, Qi F, Wu B, Sinha U, et al: Interleukin 6 and interleukin 8 as potential biomarkers for oral cavity and oropharyngeal squamous cell carcinoma. Arch Otolaryngol Head Neck Surg 130: 929-935, 2004.

25. Malhotra R, Patel V, Vaqué JP, Gutkind JS and Rusling JF: Ultrasensitive electrochemical immunosensor for oral cancer biomarker IL-6 using carbon nanotube forest electrodes and multilabel amplification. Anal Chem 82: 3118-3123, 2010

26. Ferreira FO, Ribeiro FL, Batista AC, Leles CR, de Cássia Gonçalves Alencar R and Silva TA: Association of CCL2 with lymph node metastasis and macrophage infiltration in oral cavity and lip squamous cell carcinoma. Tumour Biol 29: 114-121, 2008.

27. Sriuranpong V, Park JI, Amornphimoltham P, Patel V, Nelkin BD and Gutkind JS: Epidermal growth factor receptor-independent constitutive activation of STAT3 in head and neck squamous cell carcinoma is mediated by the autocrine/paracrine stimulation of the interleukin 6/gp130 cytokine system. Cancer Res 63 2948-2956, 2003.
28. Tanabe H, Takada Y, Minegishi D, Kurematsu M, Masui T and Mizusawa H: Cell line individualization by STR multiplex system in the cell bank found cross contamination between ECV304 and EJ-1/T24. Tissue Cult Res Commun 18: 329-338, 1999.

29. Zhao M, Sano D, Pickering CR, Jasser SA, Henderson YC, Clayman GL, Sturgis EM, Ow TJ, Lotan R, Carey TE, et al: Assembly and initial characterization of a panel of 85 genomically validated cell lines from diverse head and neck tumor sites. Clin Cancer Res 17: 7248-7264, 2011.

30. Razidlo GL, Burton KM and McNiven MA: Interleukin-6 promotes pancreatic cancer cell migration by rapidly activating the small GTPase CDC42. J Biol Chem 293: 11143-11153, 2018.

31. Liu T, Ma H, Shi W, Duan J, Wang Y, Zhang C, Li C, Lin J, Li S, Lv J, et al: Inhibition of STAT3 signaling pathway by ursolic acid suppresses growth of hepatocellular carcinoma. Int J Oncol 51: $555-562,2017$

32. Misumi Y, Okamoto H, Sasaki J, Masuda N, Ishii M, Shimokawa T, Hosomi Y, Okuma Y, Nagamata M, Ogura T, et al: Phase I/II study of induction chemotherapy using carboplatin plus irinotecan and sequential thoracic radiotherapy (TRT) for elderly patients with limited-disease small-cell lung cancer (LD-SCLC): TORG 0604. BMC Cancer 17: 377-385, 2017.

33. Tominaga H, Setoguchi T, Shimada H, Nagano S, Sasaki H, Ishidou Y, Sato M, Mizuno K, Inoue H and Komiya S: Prognostic factors in patients with skeletal-related events at non-small-cell lung cancer diagnosis. Mol Clin Oncol 7: 897-902, 2017.

34. Johnson DE, O'Keefe RA and Grandis JR: Targeting the IL-6/JAK/STAT3 signalling axis in cancer. Nat Rev Clin Oncol 15: 234-248, 2018.

35. Song Y, Zheng S, Wang J, Long H, Fang L, Wang G, Li Z, Que T, Liu Y, Li Y, et al: Hypoxia-induced PLOD2 promotes proliferation, migration and invasion via PI3K/Akt signaling in glioma. Oncotarget 8: 41947-41962, 2017.

36. Hu Y, He MY, Zhu LF, Yang CC, Zhou ML, Wang Q, Zhang W, Zheng YY, Wang DM, Xu ZQ, et al: Tumor-associated macrophages correlate with the clinicopathological features and poor outcomes via inducing epithelial to mesenchymal transition in oral squamous cell carcinoma. J Exp Clin Cancer Res 35: 12-30, 2016.

37. Komohara Y, Jinushi M and Takeya M: Clinical significance of macrophage heterogeneity in human malignant tumors. Cancer Sci 105: 1-8, 2014

38. Mori K, Hiroi M, Shimada J and Ohmori Y: Infiltration of $\mathrm{m} 2$ tumor-associated macrophages in oral squamous cell carcinoma correlates with tumor malignancy. Cancers (Basel) 3: 3726-3739, 2011.

39. Pastushenko I, Brisebarre A, Sifrim A, Fioramonti M, Revenco T, Boumahdi S, Van Keymeulen A, Brown D, Moers V, Lemaire $\mathrm{S}$, et al: Identification of the tumour transition states occurring during EMT. Nature 556: 463-468, 2018.

This work is licensed under a Creative Commons Attribution-NonCommercial-NoDerivatives 4.0 International (CC BY-NC-ND 4.0) License. 